\title{
Kinematic analysis of wrist motion during simulated colonoscopy in first-year gastroenterology fellows
}

\author{
Authors \\ Shiva K Ratuapli ${ }^{1}$, Kevin C Ruff ${ }^{1}$, Francisco C Ramirez ${ }^{1}$, Qing Wu², Deepika Mohankumar ${ }^{3}$, Marco Santello ${ }^{3}$, \\ David E Fleischer $^{1}$ \\ Institutions \\ ${ }^{1}$ Division of Gastroenterology, Mayo Clinic Arizona \\ ${ }^{2}$ Division of Health Sciences and Research, Mayo Clinic Arizona \\ ${ }^{3}$ School of Biological and Health Systems Engineering, Arizona State University, Tempe, Arizona
}

Bibliography

DOI http://dx.doi.org/

10.1055/s-0034-1393061

Published online: 5.11.2015

Endoscopy International Open

2015; 03: E621-E626

(c) Georg Thieme Verlag KG

Stuttgart · New York

E-ISSN 2196-9736

\section{Corresponding Author:}

\section{Kevin C Ruff MD}

Division of Gastroenterology

13400 East Shea Boulevard

Scottsdale, AZ 85259

Phone: 480-301-6990

Fax: 480-301-6737

ruff.kevin@mayo.edu
Background and study aims: Gastroenterology trainees acquire skill and proficiency in performing colonoscopies at different rates. The cause for heterogeneous competency among the trainees is unclear. Kinematic analysis of the wrist joint while performing colonoscopy can objectively assess the variation in wrist motion. Our objective was to test the hypothesis that the time spent by the trainees in extreme ranges of wrist motion will decrease as the trainees advance through the fellowship year.

Subjects and methods: Five first-year gastroenterology fellows were prospectively studied at four intervals while performing simulated colonoscopies. The setting was an endoscopy simulation laboratory at a tertiary care center. Kinematic assessment of wrist motion was done using a magnetic position/orientation tracker held in place by a custom-made arm sleeve and hand glove. The main outcome measure was time spent performing each of four ranges of wrist motion (mid, cen-

\section{Introduction}

\section{$\nabla$}

Gastroenterology fellowship provides the initial exposure to endoscopy with continued training over a period of 3 years for the majority of gastroenterology trainees. Skill and competency in performing endoscopy varies among individuals, and proficiency in performing endoscopy has been reported to increase at different rates [1] among individual trainees. Several factors have been used to measure competency, including cecal intubation rate, colonoscopy completion rate, and adenoma detection rate $[2,3]$. The cause of varied competency among trainees remains unclear.

Kinematics is a branch of mechanics that describes the motion of individuals, as well as groups of objects, without consideration of the causes of motion $[4,5]$. This discipline has been used to assess technical performance of trainees by measuring upper extremity joint movements ter, extreme, and out) for each wrist degree of freedom (pronation/supination, flexion/extension, and adduction/abduction).

Results: There were no statistically significant differences in the time spent for wrist movements across the three degrees of freedom throughout the study period. However, fellows spent significantly less time in extreme range $(1.47 \pm 0.34 \mathrm{~min}$ vs. $2.44 \pm 0.34 \mathrm{~min}, P=0.004)$ and center range ( $1.02 \pm 0.34 \mathrm{~min}$ vs $1.9 \pm 0.34 \mathrm{~min}, P=0.01$ ) at the end of the study compared to the baseline evaluation. The study was limited by the small number of subjects and performance of colonoscopies on a simulator rather than live patients.

Conclusions: Gastroenterology trainees alter the time spent at the extreme range of wrist motion as they advance through training. Endoscopy training during the first 10 months of fellowship may have beneficial effects on learning ergonomically correct motion patterns.

in surgical specialties using various models $[6,7]$ Because the wrist is a dominant joint involved in performing endoscopies, it is plausible that kinematic analysis of the wrist joint might identify patterns of joint movements associated with improved competency as a trainee advances through the training.

Currently, there are no data regarding the time spent by trainees in various ranges of wrist movement or its correlation with efficacy and quality of performing endoscopy as a function of practice. To address the aforementioned issues, we designed the current pilot study to test the hypothesis that the time spent in different ranges of wrist motion will change as a trainee advances through the fellowship year. 


\section{Subjects and methods}

$\nabla$

Five first-year gastroenterology fellows (4 males, 1 female; all right-handed with one male being ambidexterous) from the Mayo Clinic Arizona and Banner Good Samaritan VA medical center participated in the study. The subjects were aged 30 to 35 years. None of the fellows had prior endoscopic experience before beginning their fellowship training. Prior to the study, all the fellows attended the American Society for Gastroenterology first year fellows' course, where in they received approximately 4 hours of hands-on endoscopic training on pig models. Wrist motion data were collected across four sessions, one baseline and three follow-ups. These measurements were made when the fellows performed simulated colonoscopies using the Simbionix GI Mentor Endoscopy simulator (GI Mentor ${ }^{\mathrm{TM}}$, Simbionix, USA, Cleveland, $\mathrm{OH}$ ) ( Fig. 1). The fellows had no experience in performing endoscopy when baseline wrist range-of-motion data were collected in July 2012. The follow-up data collection sessions were conducted in October 2012, February 2012, and May 2013. During this period, the fellows performed standard colonoscopies on live patients as a part of their fellowship training. The study was approved by the institutional review boards (IRB \# 09-000450) of Mayo Clinic Arizona and Arizona State University.

\section{Wrist motion measurements}

The measurements of wrist motion were done using a magnetic position/orientation tracker (Polhemus Fastrak, Colchester, VT; $0.075 \mathrm{~mm}$ and $0.05^{\circ}$ resolution), as previously described by our group [8]. This system provides real-time 3 Degree-of-Freedom (DoF) tracking that is reliable and accurate and uses $\mathrm{A} / \mathrm{C}$ magnetics to detect the position/orientation of an object. The set-up consists of a System Electronics Unit (SEU), a power supply, one receiver, and trackers (one on the elbow and one on the hand). A custom-made arm sleeve and hand glove was used to hold the position/orientation trackers in place and maintain appropriate tracker location while the trainee performed simulated colonoscopy ( $\bullet$ Fig. 2) [8]. The arm sleeve held the transmitter over the lateral epicondyle of the right elbow joint, while the hand glove secured the receiver over the dorsal surface of the right hand, allowing measurement of changes in position of the right wrist joint. These wrist motion data were collected through a serial port at a sampling frequency of $120 \mathrm{~Hz}$. The trainees were then asked to hold the right wrist joint in a "neutral" position with the right hand held in the parasagittal plane so that the thumb pointed toward the ceiling and the little finger pointed toward the floor. This "neutral" position was considered the center point of the wrist motion ranges. The wrist was then held in six extreme positions (pronation, supination, flexion, extension, abduction and adduction) and angular data were recorded for $5 \mathrm{sec}-$ onds each in these positions. Trainees then performed simulated colonoscopies and the wrist motion data were continuously recorded during these procedures. At every data collection time point, each trainee performed two simulated colonoscopies, one being easy and the other being difficult as determined by the simulator software. The order of the procedures was counterbalanced and the trainees completed each procedure in 10 minutes or less. Prior to the actual recording, subjects practiced a case study from the first module of the simulator to accustom themselves with the equipment and the procedure. This was uniformly performed during the four data collection time points. During all trials, the experimenter made sure that the transmitter and receiver did not move from the initial locations on the elbow

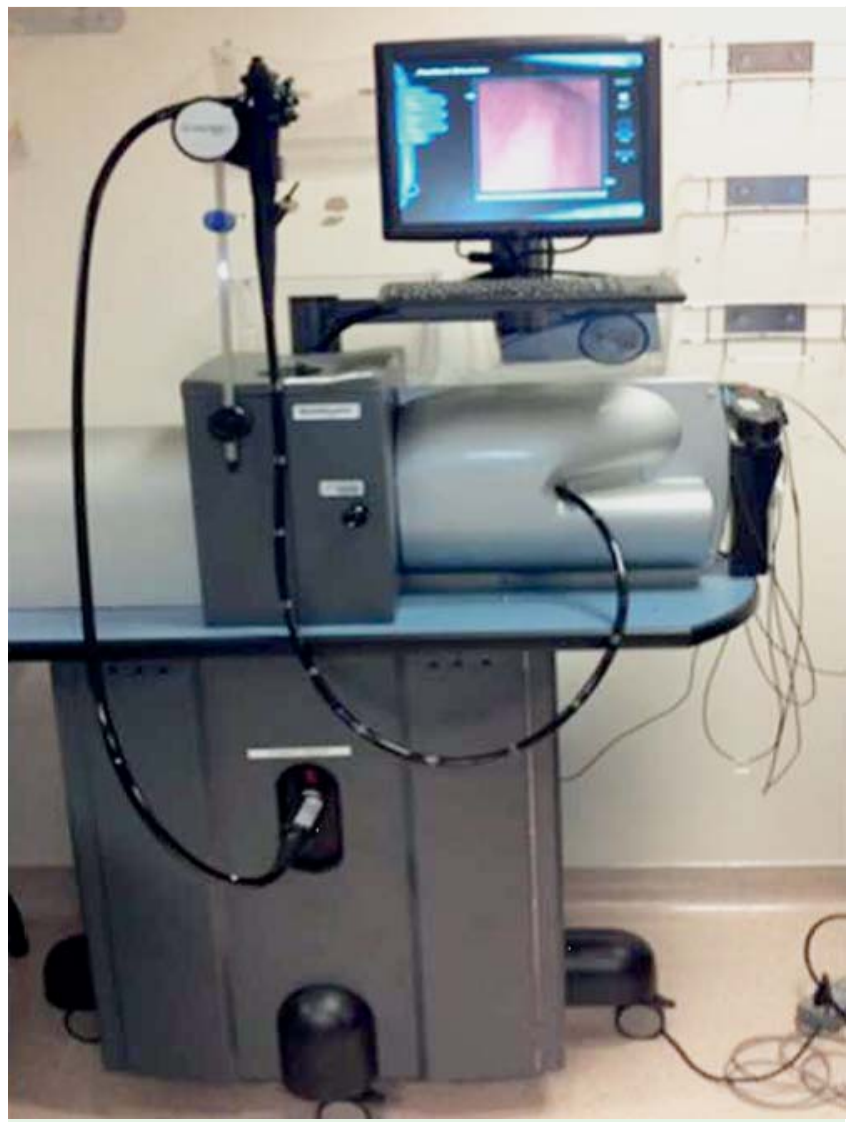

Fig. 1 Symbionix GI Mentor Simulator

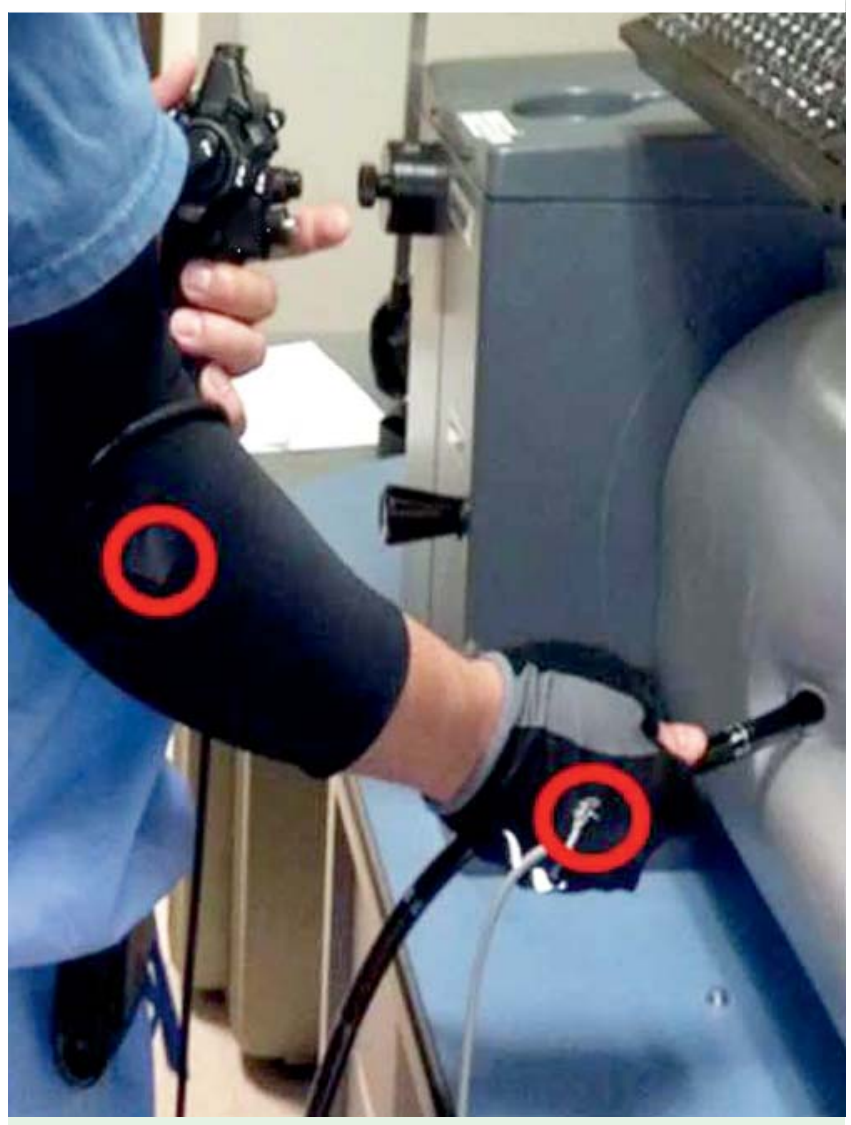

Fig. 2 Subject with magnetic position trackers held in place by custommade arm sleeve and glove. 


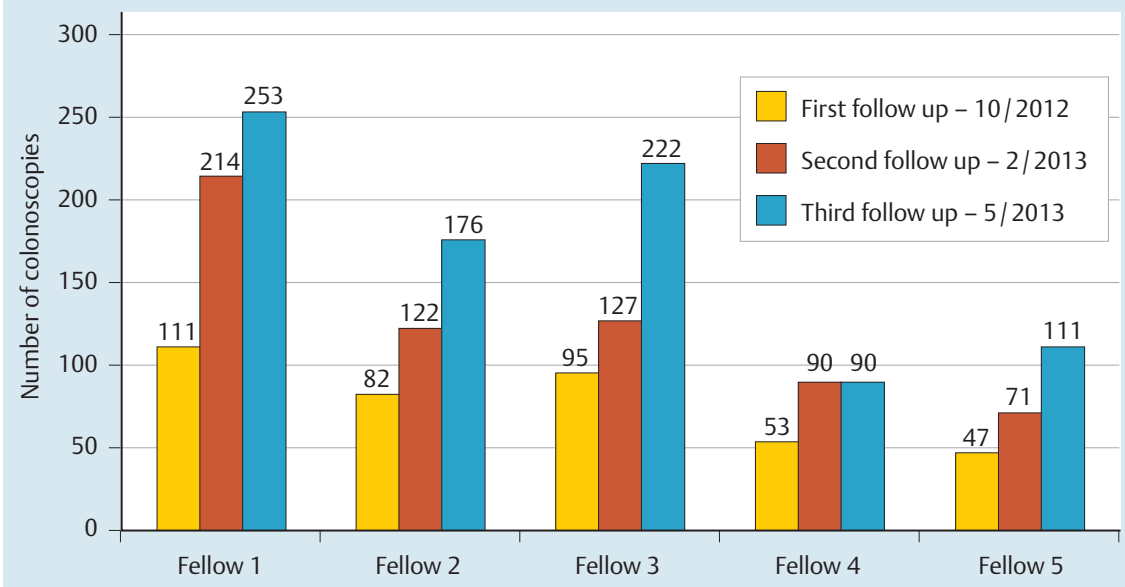

Fig. 3 Cumulative number of live-patient colonoscopies performed by subjects prior to each recording session.

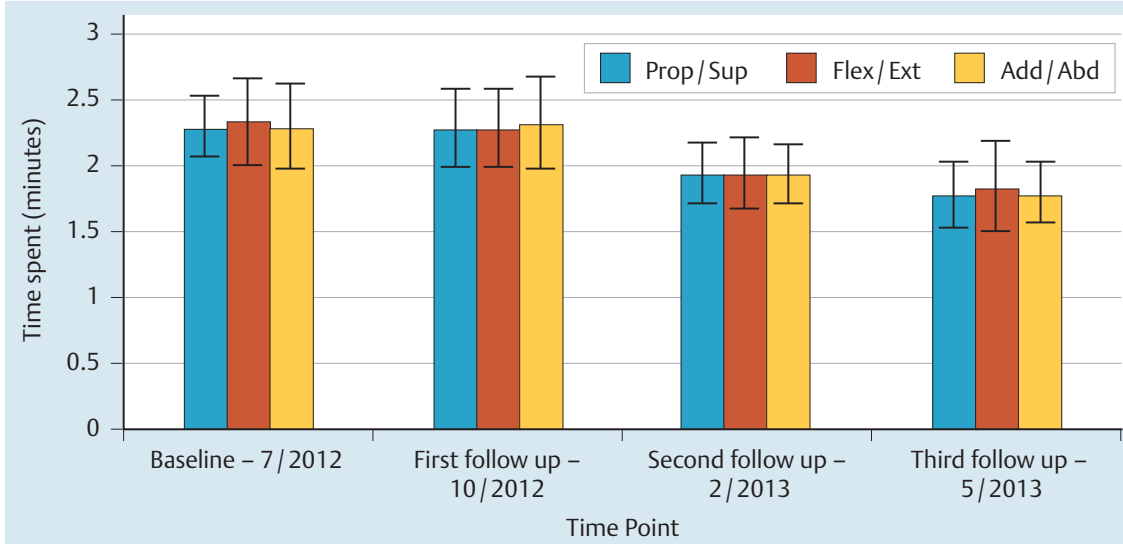

Fig.4 Distribution of time spent in various wrist positions by fellows as a group at four time points.

and hand. If any movement was detected, the recording was stopped and redone again to ensure measurement accuracy of wrist joint kinematics.

\section{Statistical analysis}

The wrist motion data were analyzed using custom software (Matlab, The MathWorks, Natick, MA). The raw data were filtered with a low-pass Butterworth filter ( $8 \mathrm{~Hz}$ cut-off frequency) to remove any high-frequency noise. The entire time series was then split into four different ranges, namely "center," "mid," "extreme," and "out" (see Mohankumar et al., 2014, for details)[8]. Briefly, the time series of joint angles recorded for each procedure was binned in $0.1^{\circ}$ increments for each DoF. The range definitions were as follows. Wrist angular data $+\mid-10 \%$ from the neutral point were defined as the "center range." Data $+/-20 \%$ relative to the center range were defined as the "mid range." Data $+\mid-20 \%$ minus the extreme postures were defined as the "extreme range." Any data that were out of this range were "out of range." Once these ranges were identified, the time spent (in minutes) in each of these ranges was calculated.

Statistical analysis was based on a mixed-effect model. The fixed effect in the mixed model included four time points (baseline vs. follow up $1-3$ ), four ranges (center vs. mid vs. extreme vs. out), two procedures (easy vs. difficult), and three wrist movement DoF (pronation/supination vs. flexion/extension vs. abduction/ adduction). The interaction term of range and time points was analyzed and included in the model. Other interaction terms were not significant and were dropped from the final model. The random effects allow covariance to vary across subjects. Post hoc pairwise comparisons were conducted for subgroups of time point and range combinations. All statistical analyses were performed using SAS software version 9.3 (SAS Institute Inc, Cary, NC). $P$ values $\leq 0.05$ were considered statistically significant. Data are presented as mean \pm standard error mean (SEM).

\section{Results \\ $\nabla$}

Wrist motion data were collected from five first-year gastroenterology fellows ( 4 males, 1 female). The baseline data were collected when they had no prior colonoscopy training. The follow-up data were recorded when the number of colonoscopies in live patients performed by individual fellows ranged from 90 to 253 over a period of 10 months ( $\bullet$ Fig. 3).

\section{Relationship between duration of training and wrist position}

The time spent by trainees in wrist pronation/supination, flexion/extension, and adduction/abduction did not differ significantly $(P=0.99)$ between baseline recording and the subsequent three follow-up recordings ( $\mathbf{F i g} .4$ ). Fellows as a group spent slightly more time in flexion/extension compared with the other two wrist positions both at baseline $(2.33 \pm 0.33$ mins vs $2.29 \pm$ 0.23 mins \& $2.29 \pm 0.32$ mins ) and the third follow-up session ( $1.83 \pm 0.34$ mins vs $1.78 \pm 0.25$ mins \& $1.78 \pm 0.23 \mathrm{mins}$ ), but these differences were not statistically significant. 
Table 1 Comparison of time spent by fellows in various ranges of wrist motion between baseline and follow-up recordings

\begin{tabular}{|c|c|c|c|c|c|c|}
\hline Time Point (1) & Range (1) & Time Point (2) & Range (2) & Mean Difference (1-2) & Standard Error & $P$-value \\
\hline \multicolumn{7}{|c|}{ Third follow up compared to baseline } \\
\hline 3 rd follow up & Out & Baseline & Out & 0.2 & 0.34 & 0.56 \\
\hline 3 rd follow up & Extreme & Baseline & Extreme & -0.97 & 0.34 & 0.004 \\
\hline 3 rd follow up & Mid & Baseline & Mid & -0.37 & 0.34 & 0.27 \\
\hline 3 rd follow up & Center & Baseline & Center & -0.88 & 0.34 & 0.01 \\
\hline \multicolumn{7}{|c|}{ Second follow up compared to baseline } \\
\hline 2nd follow up & Out & Baseline & Out & 0.64 & 0.34 & 0.06 \\
\hline 2nd follow up & Extreme & Baseline & Extreme & -0.49 & 0.34 & 0.15 \\
\hline 2nd follow up & Mid & Baseline & Mid & -0.9 & 0.34 & 0.008 \\
\hline 2nd follow up & Center & Baseline & Center & -0.75 & 0.34 & 0.03 \\
\hline \multicolumn{7}{|c|}{ First follow up compared to baseline } \\
\hline 1st follow up & Out & Baseline & Out & 0.21 & 0.34 & 0.54 \\
\hline 1st follow up & Extreme & Baseline & Extreme & -0.5 & 0.34 & 0.14 \\
\hline 1st follow up & Mid & Baseline & Mid & 0.14 & 0.34 & 0.69 \\
\hline 1st follow up & Center & Baseline & Center & 0.096 & 0.34 & 0.78 \\
\hline
\end{tabular}

\section{Relationship between duration of training and wrist} range of motion

Comparison of various predefined ranges of wrist motion (i.e., center, mid, extreme, out) between the third follow-up and baseline recordings showed that there were significant differences in the time spent by the fellows as a group in specific ranges of motion $(\bullet$ Table 1$)$. By the end of the study period, fellows spent significantly less time in the "extreme" $(1.47 \pm 0.34 \mathrm{~min}$ vs $2.44 \pm$ $0.34 \mathrm{~min}, P=0.004)$ and "center" ranges $(1.02 \pm 0.34 \mathrm{~min}$ vs $1.9 \pm$ $0.34 \mathrm{~min}, P=0.01$ ) compared with the baseline recordings ( $\bullet$ Fig.5). Similarly, when the second follow up was compared to the baseline, fellows spent significantly less time in the "mid" and "center" ranges of motion ( $\bullet$ Table $\mathbf{1})$. However, there were no significant differences in the time spent at any of the ranges of motion when first follow up was compared with baseline.

\section{Discussion}

$\nabla$

Current objective measures of performing colonoscopy and training gastroenterology fellows in performing colonoscopy are poor and not standardized. Our experiment is the first study to explore the possibility of using kinematics for direct objective assessment of the time spent in various ranges of motion by the right wrist joint, which is primarily involved in torque and control of the shaft during colonoscopy. Our results show that as trainees advance through the first year of training, the pattern and range of wrist motion changes significantly. Trainees spent significantly less time in the "center" and "extreme" ranges of motion of the wrist joint as they advanced through the year and performed more procedures. These findings may have implications in the training of gastroenterology fellows and provide insight into mechanisms of varied competency among gastroenterology fellows. Further research in this field may potentially identify specific joint motions associated with efficient endoscopic maneuvers, and these skills can subsequently be taught to trainees, using real-time kinematic motion feedback.

Over the past decade, some studies have attempted to evaluate the forces exerted during colonoscopy using hand and thumb force measuring devices [9-12] as well as electromyography (EMG) [12] of the forearm muscles. Shergill et al [12] found that pinch forces of the right thumb, and EMG activity of forearm muscles were highest during insertion of the colonoscope into the right and left colon. Using a colonoscopy force measuring device attached to the colonoscope, Appleyard et al [9] showed that the range of push forces exerted during colonoscopy was wide. Korman et al [11] showed that push/pull and torque forces varied among the endoscopists, and they could be grouped by the force application patterns. Recently, Obstein et al [13] used a colonoscope with electromagnetic sensors for indirect kinematic analysis of scope movement in a colon model, and showed that the pattern varied consistently in gastroenterology fellows by their year of training.

In contrast to the previous studies, this is the first study to perform a direct kinematic analysis of the wrist joint, rather than to assess the force exerted on the colonoscope or indirectly measure the transmitted movements of the colonoscope shaft. This method may provide complementary information about the biomechanics of colonoscopy procedures and may provide reliable objective measures of performing a quality colonoscopy. Our results support the study by Obstein et al [13], who assessed wrist motion indirectly by measuring the curvature of the endoscope, tip angulation, and the absolute roll of the endoscope. They showed that the faculty and third-year fellows had better performance

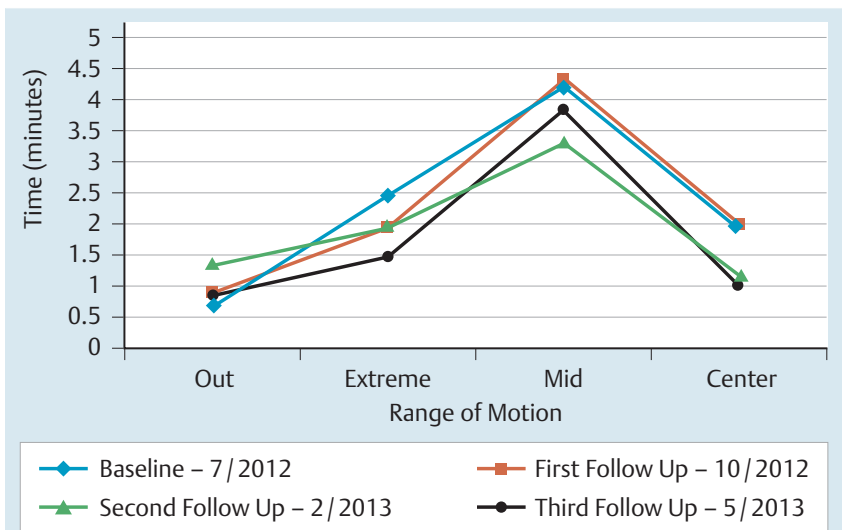

Fig. 5 Distribution of time spent in various ranges of wrist motion by fellows as a group at four time points 


\begin{tabular}{|llll|}
\hline Range of Motion & $\begin{array}{l}\text { Gastroenterology trainees } \\
\text { baseline measurement } \\
(\mathbf{m i n s})\end{array}$ & $\begin{array}{l}\text { Gastroenterology trainees } \\
\text { third follow-up measure- } \\
\mathbf{( n = 5 )}(\text { Mean } \pm \text { SD) }\end{array}$ & $\begin{array}{l}\text { Experienced endoscopists } \\
(\mathbf{m}=\mathbf{5})(\mathbf{m} \text { (Mean } \pm \text { SD) }\end{array}$ \\
\hline Out & $0.67 \pm 0.93$ & $0.87 \pm 1.1$ & $1 \pm 1.67$ \\
\hline Extreme & $2.44 \pm 1.58$ & $1.47 \pm 1.09$ & $1.75 \pm 1.32$ \\
\hline Mid & $4.21 \pm 1.47$ & $3.83 \pm 1.8$ & $3.98 \pm 1.83$ \\
\hline Center & $1.9 \pm 1.6$ & $1.02 \pm 1.06$ & $2.31 \pm 1.7$ \\
\hline
\end{tabular}

Table 2 Comparison between time spent by fellows in various ranges of wrist motion at baseline measurement versus that for experienced endoscopists in a previous study [8] than the first-year fellows. However, unlike our study, they did not assess kinematics in the same subject over a period of time. The exact reason for the variation in wrist motion during different time points of a fellows' training is unclear. We speculate that as trainees advance through fellowship, they become increasingly adept at intubating the left and right colon, which are traditionally a difficult part of the colon to navigate, and may require less extreme ranges of motion of the right wrist joint. The study by Shergill et al [12], which showed that increased muscle forces were necessary to intubate the left and right colons, supports this hypothesis. Hence, toward the end of the training year, with increased competence in performing colonoscopies, the extreme range of wrist motion may be significantly lower, when compared with the beginning of the training year. In contrast, the reason for reduced time spent in the center range remains unclear. We can only speculate that as the trainees advanced in their motor skills, the overall body posture and altered utilization of the left hand to hold the head of the colonoscope might have affected the center range more than the other ranges of motion. Because our study focuses on the wrist motion of gastroenterology fellows during only their first year of training, it is unknown if the observed alterations in wrist motion have any implications on overuse injuries as the trainees advance in their career as practicing gastroenterologists. Although we did not compare the wrist motion of trainees with that of experienced endoscopists in this study, published data from a previous study[8] by our group shows that at baseline measurement, the trainees spent more time in extreme range of wrist motion than did experienced gastroenterologists $(2.44 \pm 1.58$ min vs $1.75 \pm 1.32)$, as shown in $\bullet$ Table 2. However, we did not perform statistical analysis of the comparison, as the simulated cases used to perform colonoscopies were different between the two groups of subjects.

There are some limitations to our study. Colonoscopies were performed on an endoscopy simulator, rather than on actual patients. However, simulators have been used in training [14] for assessing the competency of gastroenterology fellows [15], are moderately realistic compared to human colonoscopy [16], can predictably reproduce the difficulty of an exam, and can differentiate experienced from novice endoscopists [17]. Performing these studies during actual patient colonoscopies would be of value and will be considered for future studies but is not reproducible among study subjects as can be done with simulators. The sample size was small with five subjects performing 40 simulated colonoscopies; however, we had the advantage of studying the individual subjects on four separate occasions over a 10month period. The range of wrist motion defined in our study is not standardized; however, we attempted to define the range of wrist motion a priori, based on the physiologic range of movement of the wrist for everyday tasks.

In summary, kinematic analysis of the wrist joint provides a means of assessing normal range of wrist motion during colonos- copy, and may yield objective measures for training gastroenterology fellows. Trainees seem to change the pattern in the range of their wrist motion as they advance though training. Even though these experiments were done on a small number of study subjects, our study is a proof of principle that this novel technique can be used to assess wrist motion during endoscopy. Further research in this area may provide insight into improving the technique of colonoscopy and prevent musculoskeletal injuries during endoscopy.

Competing interest: Grant Support: Partial grant support from an ASU-Mayo Innovation Technology Seed Grant.

\section{Acknowledgements \\ $\nabla$}

The authors thank Simbionix USA, Cleveland, OH, for providing the simulator for the study. The authors also thank gastroenterology fellows Dr. Mary A. Atia, Dr. Bobby R. Kakati, Dr. Darren J. Andrade, and Dr. Michael Foley for participating in the study as research subjects.

\section{References}

1 Marshall JB. Technical proficiency of trainees performing colonoscopy: a learning curve. Gastrointest Endosc 1995; 42: 287-291

2 Cass OW, Freeman ML, Peine CJ et al. Objective evaluation of endoscopy skills during training. Ann Intern Med 1993; 118: 40-44

3 Peters SL, Hasan AG, Jacobson NB et al. Level of fellowship training increases adenoma detection rates. Clin Gastroenterol Hepatol 2010; 8: 439-442

4 Beggs JS. Kinematics. Washington: Hemisphere Pub. Corp; 1983: xvi $223 \mathrm{p}$

5 Bottema O, Roth B. Theoretical kinematics. New York: Dover Publications; 1990: xiv $558 \mathrm{p}$

6 Leong JJ, Leff DR, Das A et al. Validation of orthopaedic bench models for trauma surgery. J Bone Joint Surg Br 2008; 90: 958 - 965

7 Saleh GM, Lindfield D, Sim D et al. Kinematic analysis of surgical dexterity in intraocular surgery. Arch Ophthalmol 2009; 127: 758-762

8 Mohankumar D, Garner H, Ruff $K$ et al. Characterization of right wrist posture during simulated colonoscopy: an application of kinematic analysis to the study of endoscopic maneuvers. Gastrointest Endosc 2014; 79: 480-489

9 Appleyard MN, Mosse CA, Mills TN et al. The measurement of forces exerted during colonoscopy. Gastrointest Endosc 2000; 52: 237-240

10 Dogramadzi S, Virk GS, Bell GD et al. Recording forces exerted on the bowel wall during colonoscopy: in vitro evaluation. Int J Med Robot 2005; $1: 89-97$

11 Korman LY, Egorov V, Tsuryupa $S$ et al. Characterization of forces applied by endoscopists during colonoscopy by using a wireless colonoscopy force monitor. Gastrointest Endosc 2010; 71: 327-334

12 Shergill AK, Asundi KR, Barr A et al. Pinch force and forearm-muscle load during routine colonoscopy: a pilot study. Gastrointest Endosc 2009; 69: $142-146$

13 Obstein KL, Patil VD, Jayender J et al. Evaluation of colonoscopy technical skill levels by use of an objective kinematic-based system. Gastrointest Endosc 2011; 73: 315-321, 321 e311 
14 Coderre S, Anderson J, Rostom A et al. Training the endoscopy trainer: from general principles to specific concepts. Can J Gastroenterol 2010; 24: 700 - 704

15 Sedlack RE, Kolars JC. Computer simulator training enhances the competency of gastroenterology fellows at colonoscopy: results of a pilot study. Am J Gastroenterol 2004; 99: 33-37
16 Hill A, Horswill MS, Plooy AM et al. Assessing the realism of colonoscopy simulation: the development of an instrument and systematic comparison of 4 simulators. Gastrointest Endosc 2012; 75: 631 - 640

17 Ansell J, Mason J, Warren $N$ et al. Systematic review of validity testing in colonoscopy simulation. Surg Endosc 2012; 26: 3040 - 3052 